Pacific Journal of Mathematics

ON THE ISOLATION OF ZEROES OF AN ANALYTIC 


\title{
ON THE ISOLATION OF ZEROES OF AN ANALYTIC FUNCTION
}

\author{
DOUGLAS S. BRIDGES
}

The purpose of this paper is to describe extensions of the work of Errett Bishop on the location of zeroes of complex-valued analytic functions. The main result deals with the number of zeroes of an analytic function $f$ near the boundary of a closed disc well contained in the domain of $f$. A particular consequence of this result is the following theorem.

Let $f$ be analytic and not identically zero on a connected open subset $U$ of $C, K$ a compact set well contained in $U$, and $\varepsilon>0$. Then either inf $\{|f(z)|: z \in K\}>0$ or there exist finitely many points $z_{1}, \cdots, z_{n}$ of $U$ and an analytic function $g$ on $U$ such that

$$
f(z)=\left(z-z_{1}\right) \cdots\left(z-z_{n}\right) g(z) \quad(z \in U),
$$

$\inf \{|g(z)|: z \in K\}>0$ and $d\left(z_{k}, K\right)<\varepsilon$ for each $k$.

The paper is written entirely within the framework of Bishop's constructive mathematics.

As Bishop has remarked [1, p. 112], the constructive development of the elementary theory of analytic functions of one complex variable presents comparatively few serious difficulties. One topic in which difficulties do arise, however, is that of location of zeroes of an analytic function. In this paper, we derive several results which apply and strengthen those obtained by Bishop [1, Ch. 5, §5]. and which present different constructive facets of the classical theorem that the zeroes of an analytic function are isolated.

For the reader who knows little about the spirit or aims of modern constructive mathematics, we recommend Allan Calder's recent article in Scientific American [4]. The necessary technical background in constructive analysis is found in [1] and [2]; in particular, we shall assume knowledge of Chapter 5 of [1]. However, it is expedient to recall here two definitions from that chapter.

A compact subset $K$ of an open set $U$ in $C$ is well contained in $U$ if there exists $r>0$ such that $\{z \in C: d(z, K) \leqq r\} \subset U$; in which case we write $K \subset \subset U$. If $K$ is a compact subset of $C$, then a border for $K$ is a compact subset $B$ of $K$ such that $\bar{B}(z, d(z, B)) \subset$ $K$ for each $z$ in $K$. (We write $\bar{B}(z, r)$ for the closed disc, and $B(z, r)$ for the open disc, of center $z$ and radius $r$.)

We should also note that a complex-valued function $f$ is not identically zero on a set $A$ if there exists $z$ in $A$ with $|f(z)|>0$; 
and that $f$ is nonvanishing, or does not vanish, on $A$ if $|f(z)|>0$ for each $z$ in $A$.

1. Bishop's main results on the location and isolation of zeroes are contained in the Corollary on p. 135, and Theorem 7 on p. 138, of [1], and are summarised as follows.

THEOREM 1. Let $K$ be a compact subset of $C, B$ a border for $K$, and $f$ a differentiable function on $K$ with $\inf \{|f(z)|: z \in B\}>0$. Then either $\inf \{|f(z)|: z \in K\}>0$ or there exist finitely many points $z_{1}, \cdots, z_{n}$ of $K$, a differentiable function $g$ on $K$, and $c>0$ such that, for each $z$ in $K$,

$$
f(z)=\left(z-z_{1}\right) \cdots\left(z-z_{n}\right) g(z)
$$

and $|g(z)| \geqq c$.

Although this theorem is strong enough to yield the Fundamental Theorem of Algebra as a consequence [1, p. 140, Thm. 8], it conveys no information unless the border $B$ of $K$ is strict, in the sense that $d(\zeta, B)>0$ for some $\zeta$ in $K$ (in which case we say that $K$ is strictly bordered). In particular, circles in the complex plane are not strictly bordered, but we often want to know whether or not $f$ is bounded away from zero on certain circles in its domain. (This question is particularly relevant when we are trying to integrate $f^{\prime} / f$ round circles.) In fact, we might expect that, if $f$ is analytic and not identically zero on a connected open set $U$, then it is bounded away from zero on all but finitely many circles in any given disc well contained in $U$.

On the other hand, even if the compact set $K$ has a strict border $B$, it is easy to see that we cannot expect to decide whether or not the modulus of a differentiable function $f$ on $K$ has positive infimum on $B$ : consider the example where $K=\bar{B}(0,1), B=\{z \in C$ : $|z|=1$ ) and $f$ is the function $z \rightarrow z-\zeta$, $\zeta$ being a real number for which the alternative " $\zeta>1$ or $\zeta \leqq 1$ " is undecided.

This last example also illustrates why we cannot expect to locate precisely the zeroes of an analytic function which lie in a given compact set. However, it is reasonable to expect that, if $f$ is analytic and not identically zero on a connected open set $U$, and $K$ is a compact set well contained in $U$, then we can find all the zeroes (if any) of $f$ that lie within a distance $\varepsilon$ of $K$ for some suitable $\varepsilon>0$.

In what follows, we shall show that the above expectations are fulfilled. As a bonus, we shall obtain a proof that, if $f$ is analytic on an open set $U$ and nonvanishing on a compact set $K$ well con- 
tained in $U$, then $1 / f$ is bounded away from 0 on $K$. This last result is particularly satisfactory in view of our present inability to compute a positive lower bound for a continuous mapping of a compact interval into the positive reals [3].

2. The cornerstone of our paper is Theorem 2 below, which, applied to a differentiable function $f$ on $\bar{B}(0,1)$ with $|f(0)|>0$, enables us to estimate the number of zeroes of $f$ inside, but near to, a circle of center 0 and any given positive radius $\rho<1$. More precisely, it enables us to find circles, of center 0 and radius arbitrarily close to $\rho$, on which $f$ is bounded away from zero.

The proof of this theorem is sufficiently complicated to warrant a few remarks on its origin. Classically, a function $f$ that is differentiable and not identically zero on $\bar{B}(0,1)$ has at most finitely many zeroes in $\bar{B}(0, \rho)$ whenever $0<\rho<1$. In order to obtain a constructive analogue of this proposition, it is natural to look for an explicit a priori bound on the number of zeroes in $\bar{B}(0, \rho)$. As it happens, if $|f(0)|>0$ such a bound exists classically [7, 5.24, p. 171]; it was with that bound in mind that we were able to obtain the numerical estimates that we need for Theorem 2.

The other thing we need is an inequality which provides lower bounds for $|f(z)|$ as $z$ ranges through $\bar{B}(0,1)$. That this need can also be satisfied is the content of

Lemma 1 . Let $f$ be differentiable on the closed disc $\bar{B}(0,1)$, with $\sup \{|f(z)|:|z| \leqq 1\} \leqq 1$. Then

$$
|f(z)| \geqq \frac{|f(0)|-|z|}{1-|f(0)| \cdot|z|}
$$

for all $z$ with $|z|<1$.

Proof. Let $|\zeta|<1$, suppose that

$$
|f(\zeta)|<(|f(0)|-|\zeta|) /(1-|f(0)||\zeta|),
$$

and choose $\alpha$ so that $0<\alpha<1$ and $|f(\zeta)|>(\alpha|f(0)|-|\zeta|) \mid$ $(1-\alpha|f(0)| \cdot|\zeta|)$. As $|\alpha f(0)| \leqq \alpha<1$ [1, p 134, Proposition 5], we can apply to $\alpha f$ the argument on pages $60-61$ of [6], to obtain

$$
\left|f^{\prime}(\zeta)\right| \geqq|\alpha f(\zeta)| \geqq(|\alpha f(0)|-|\zeta|) /(1-|\alpha f(0)| \cdot|\zeta|),
$$

a contradiction. Hence, in fact, $|f(\zeta)| \geqq(|f(0)|)-|\zeta|) /(1-\mid f(0|\cdot| \zeta \mid)$.

In the following theorem, we adopt the convention that

$$
\left(1+\left(\rho^{-1}-1\right) \rho^{-1 / 0}\right)^{-1}=0 .
$$


Theorem 2. Let $\nu$ be a natural number, $0<\rho<\frac{1}{2}$, and $r_{0}, \cdots, r_{\nu}$ distinct real numbers with

$$
\left(1+\left(\rho^{-1}-1\right) \rho^{-1 / \nu}\right)^{-1} \leqq r_{k} \leqq \rho
$$

for each $k$. Let $f$ be differentiable on $\bar{B}(0,1)$, with $|f(0)|>2 \rho^{\nu+1}$ $(1-\rho)^{\nu}$ and $\sup \{|f(z)|:|z| \leqq 1\} \leqq 1$. Then

$$
\max _{k=0, \ldots, \nu} \inf \left\{|f(z)|:|z|=r_{k}\right\}>0 \text {. }
$$

Proof. If $\nu=0$ then, by Lemma 1,

$$
|f(z)| \geqq(|f(0)|-|z|) /(1-|f(0)||z|)>0 \quad(|z| \leqq \rho) .
$$

Now let $n$ be a positive integer, suppose we have proved Theorem 2 for $\nu=n-1$, and consider the case $\nu=n$. Either $2 \rho^{n} /(1-\rho)^{n-1}<$ $|f(0)|$, in which case we are through; or, as we now suppose, $|f(0)|<2 \rho^{n} /(1-\rho)^{n}$. Let

$$
\begin{aligned}
& M=2 / \prod_{k=0}^{n-1}\left(1-r_{k}\right), \\
& \alpha=\max _{k=0, \cdots, n-1} \prod_{j=0, j \neq k}^{n-1}\left(\left(1+r_{j}\right) /\left|r_{j}-r_{k}\right|\right),
\end{aligned}
$$

and define

$$
\begin{aligned}
& F\left(z, z_{0}, \cdots, z_{n-1}, a_{0}, \cdots, a_{n-1}\right) \\
& \quad=f(z)-\sum_{k=0}^{n-1} a_{k} \prod_{j=0, j \neq k}^{n-1}\left(\left(z-z_{j}\right) /\left(z_{k}-z_{j}\right)\right)
\end{aligned}
$$

for $|z| \leqq 1, a_{k} \in C,\left|z_{k}\right|=r_{k}(k=0, \cdots, n-1)$. Observe that

$$
\begin{aligned}
2 \prod_{k=0}^{n-1} r_{k}- & |f(0)| r_{n} \prod_{k=0}^{n-1}\left(1-r_{k}\right) \\
\geqq & 2\left(1+\left(\rho^{-1}-1\right) \rho^{-1 / n}\right)^{-n} \\
& -|f(0)| \rho\left(1-\left(1+\left(\rho^{-1}-1\right) \rho^{-1 / n}\right)^{-1}\right)^{n} \\
= & \left(1+\left(\rho^{-1}-1\right) \rho^{-1 / n}\right)^{-n}\left(2-|f(0)|(1-\rho)^{n} \rho^{-n}\right) \\
> & 0
\end{aligned}
$$

and that

$$
\begin{aligned}
& |f(0)| \prod_{k=0}^{n-1}\left(1-r_{k}\right)-2 \prod_{k=0}^{n} r_{k} \\
& \quad \geqq|f(0)|(1-\rho)^{n}-2 \rho^{n+1} \\
& \quad>0 .
\end{aligned}
$$

It follows from these inequalities and a simple argument using uniform continuity that there exist positive numbers $\delta, m$ such that 


$$
\frac{\left|F\left(0, z_{0}, \cdots, z_{n-1}, a_{0}, \cdots, a_{n-1}\right)\right| \prod_{k=0}^{n-1}\left(1-r_{k}\right)-2 \prod_{k=0}^{n} r_{k}}{2 \prod_{k=0}^{n-1} r_{k}-r_{n}\left|F\left(0, z_{0}, \cdots, z_{n-1}, a_{0}, \cdots, a_{n-1}\right)\right| \prod_{k=0}^{n-1}\left(1-r_{k}\right)} \geqq m
$$

whenever $\left|z_{k}\right|=r_{k},\left|a_{k}\right| \leqq \delta(k=0, \cdots, n-1)$.

Let $\omega$ be a modulus of uniform continuity for $|F|$ on the compact subset

$$
\begin{gathered}
\left\{\left(z, z_{0}, \cdots, z_{n-1}, a_{0}, \cdots, a_{n-1}\right):|z|=r_{n} ;\left|z_{k}\right|=r_{k},\left|a_{k}\right| \leqq \delta\right. \\
(k=0, \cdots, n-1)\}
\end{gathered}
$$

of $C^{2 n-1}$, where the latter is taken with the norm $|x|=\max _{k=1, \cdots, 2 n-1}\left|x_{k}\right|$. Either

$$
0<\max _{k=0, \cdots, n-1} \inf \left\{|f(z)|:|z|=r_{k}\right\}
$$

or, as we now assume,

$$
\begin{aligned}
\max _{k=0, \cdots, n-1} & \inf \left\{|f(z)|:|z|=r_{k}\right\} \\
& <\min \left(\delta, 1 / n \alpha, \omega\left(2^{-1} \operatorname{Mm} \prod_{k=0}^{n-1}\left|r_{k}-r_{n}\right|\right)\right) .
\end{aligned}
$$

Choosing $z_{0}, \cdots, z_{n-1}$ so that, for each $k,\left|z_{k}\right|=r_{k}$ and

$$
\left|f\left(z_{k}\right)\right|<\min \left(\delta, 1 / n \alpha, \omega\left(2^{-1} \operatorname{Mm} \prod_{k=0}^{n-1}\left|r_{k}-r_{n} w\right|\right)\right),
$$

define

$$
g(z)=F\left(z, z_{0}, \cdots, z_{n-1}, f\left(z_{0}\right), \cdots, f\left(z_{n-1}\right)\right) \quad(|z| \leqq 1) .
$$

Then $g$ is differentiable on $\bar{B}(0,1)$ and $g\left(z_{k}\right)=0$ for $k=0, \cdots, n-1$. By [1, p 132, Cor. 4], there exists a differentiable function $h$ on $\vec{B}(0,1)$ such that

$$
g(z)=\left(z-z_{0}\right) \cdots\left(z-z_{n-1}\right) h(z) \quad(|z| \leqq 1) .
$$

For $|z|=1$, we have

$$
\begin{aligned}
|h(z)| & \leqq|g(z)| / \prod_{k=0}^{n-1}\left(1-r_{k}\right) \\
& \leqq\left(|f(z)|+\alpha \sum_{k=0}^{n-1}\left|f\left(z_{k}\right)\right|\right) / \prod_{k=0}^{n-1}\left(1-r_{k}\right) \\
& \leqq\left(1+\alpha \sum_{k=0}^{n-1}(1 / n \alpha)\right) / \prod_{k=0}^{n-1}\left(1-r_{k}\right)=M .
\end{aligned}
$$

By the maximum principle [1, p. 134, Proposition 5], $|h(z)| \leqq M$ for each $z$ with $|z| \leqq 1$. Given $z$ with $|z|=r_{n}$, we now apply Lemma 1 to $M^{-1} h$, to obtain 


$$
\begin{aligned}
& M^{-1}|h(z)| \geqq(|h(0)|-M|z|) /(M-|z| \cdot|h(0)|) \\
& =\frac{|g(0)| \prod_{k=0}^{n-1}\left(1-r_{k}\right)-2 \prod_{k=0}^{n} r_{k}}{2 \prod_{k=0}^{n-1} r_{k}-r_{n}|g(0)| \prod_{k=0}^{n-1}\left(1-r_{k}\right)} \\
& =\frac{\left|F\left(0, z_{0}, \cdots, z_{n-1}, f\left(z_{0}\right), \cdots, f\left(z_{n-1}\right)\right)\right| \prod_{k=0}^{n-1}\left(1-r_{k}\right)-2 \prod_{k=0}^{n} r_{k}}{2 \prod_{k=0}^{n-1} r_{k}-r_{n}\left|F\left(0, z_{0}, \cdots, z_{n-1}, f\left(z_{0}\right), \cdots, f\left(z_{n-1}\right)\right)\right| \prod_{k=0}^{n-1}\left(1-r_{k}\right)} \\
& \geqq m .
\end{aligned}
$$

Hence

$$
|g(z)| \geqq|h(z)| \prod_{k=0}^{n-1}\left|r_{k}-r_{n}\right| \geqq \operatorname{Mm} \prod_{k=0}^{n-1}\left|r_{k}-r_{n}\right|
$$

and so

$$
\begin{aligned}
|f(z)| & =\left|F\left(z, z_{0}, \cdots, z_{n-1}, 0, \cdots, 0\right)\right| \\
& \geqq\left|F\left(z, z_{0}, \cdots, z_{n-1}, f\left(z_{0}\right), \cdots, f\left(z_{n-1}\right)\right)\right|-2^{-1} \operatorname{Mm} \prod_{k=0}^{n-1}\left|r_{k}-r_{n}\right| \\
& \geqq 2^{-1} \operatorname{Mm} \prod_{k=0}^{n-1}\left|r_{k}-r_{n}\right| .
\end{aligned}
$$

Thus

$$
\inf \left\{|f(z)|:|z|=r_{n}\right\} \geqq 2^{-1} \mathrm{Mm} \prod_{k=0}^{n-1}\left|r_{k}-r_{n}\right|>0 ，
$$

and our induction is complete.

3. We now use Theorem 2 to obtain a rich supply of circles on which a given analytic function, not identically zero, is bounded away from 0. In turn, this will enable us to factor out the zeroes of such a function which lie in or near a compact set well contained in a connected open subset of its domain.

LEMMA 2. Let $f$ be differentiable and not identically zero on $\bar{B}(0,1)$, and let $0<\varepsilon<\rho<\frac{1}{2}$. Then there exists $r$ such that $\rho-\varepsilon<r<\rho$ and $\inf \{|f(z)|:|z|=r\}>0$.

Proof. Let $\sum_{n=0}^{\infty} a_{n} z^{n}$ be the Taylor expansion of $f(z)$ about 0 , the series being uniformly convergent in $\bar{B}(0, \rho)$. Then there exists $n$ such that $\left|a_{n}\right|>0$. We proceed by induction on $n$.

Clearly, we lose no generality by taking $|f(z)| \leqq 1$ for all $z$ with $|z| \leqq 1$. If $n=0$, we choose a natural number $\nu$ such that $|f(0)|=\left|a_{0}\right|>2 \rho^{\nu+1} /(1-\rho)^{\nu}$ and $\left(1+\left(\rho^{-1}-1\right) \rho^{-1 / \nu}\right)^{-1}>\rho-\varepsilon$, and then 
apply Theorem 2 to $f$. On the other hand, if we have proved the lemma for any $f$ with $\max _{k=0, \cdots, n-1}\left|a_{k}\right|>0$, and we consider the case $\left|a_{n}\right|>0$, then, by the foregoing, there exists $r$ with $\rho-\varepsilon<r<\rho$ and

$$
0<m=\inf \left\{\left|\sum_{k=n}^{\infty} a_{k} z^{k-n}\right|:|z|=r\right\} .
$$

Either $0<\max _{k=0, \cdots, n-1}\left|a_{k}\right|$ or, as we may assume, $\max _{k=0, \cdots, n-1}\left|a_{k}\right|<$ $r^{n} m / 2 n$. For $|z|=r$, we now have

$$
\begin{aligned}
|f(z)| & \geqq|z|^{n}\left|\sum_{k=n}^{\infty} a_{k} z^{k-n}\right|-\sum_{k=0}^{n-1}\left|a_{k}\right||z|^{k} \\
& \geqq r^{n} m-\sum_{k=0}^{n-1}\left|a_{k}\right| \\
& >r^{n} m / 2,
\end{aligned}
$$

whence $\inf \{|f(z)|:|z|=r\} \geqq r^{n} m / 2>0$.

Lemma 3. Let $\zeta \in C, r>0$, and let $f$ be differentiable on $\bar{B}(\zeta, r)$, with $\inf \{|f(z)|:|z-\zeta|=r\}>0$. Then either inf $\{|f(z)|:|z-\zeta| \leqq r\}>0$, or there exist finitely many points $z_{1}, \cdots, z_{n}$ of $B(\zeta, r)$ and an operation $\delta: R^{+} \rightarrow R^{+}$such that $f\left(z_{k}\right)=0$ for each $k$, and $|f(z)| \geqq$ $\delta(\alpha)$ whenever $\alpha>0,|z-\zeta| \leqq r$ and $\left|z-z_{k}\right| \geqq \alpha$ for each $k$.

Proof. By Theorem 1, for each $k$ either inf $\{|f(z)|:|z-\zeta| \leqq r\}>$ 0 or there exist finitely many points $z_{1}, \cdots, z_{n}$ of $B(\zeta, r)$ and a differentiable function $g$ on $\bar{B}(\zeta, r)$ such that

$$
f(z)=\left(z-z_{1}\right) \cdots\left(z-z_{n}\right) g(z) \quad(z \in \bar{B}(\zeta, r))
$$

and $0<c=\inf \{|g(z)|:|z-\zeta| \leqq r\}$. In the latter case, we need only set $\delta(\alpha)=\alpha^{n} c$ for each $\alpha>0$.

Let $P(x)$ be a statement about the object $x$, and let $A$ be a subset of $R$. We say that $P(x)$ holds for all but finitely many $x$ in $A$ if there exist finitely many elements $x_{1}, \cdots, x_{n}$ of $R$ such that $P(x)$ is true whenever $x$ belongs to $A$ and is distinct from each of the $x_{k}$.

Proposition 1. Let $f$ be differentiable and not identically zero on $\bar{B}(0,1)$, and let $0<\rho<1$. Then $\inf \{|f(z)|:|z|=r\}>0$ for all but finitely many $r$ with $0<r<\rho$.

Proof. Choose $s$ so that $\rho<s<1$ and $\inf \{|f(z)|:|z|=s\}>0$. In view of Lemma 3 , we can assume that there exist finitely many points $z_{1}, \cdots, z_{n}$ of $B(0, s)$ and an operation $\delta: R^{+} \rightarrow R^{+}$such that 
$|f(z)| \geqq \delta(\alpha)$ whenever $\alpha>0,|z|<s$ and $\left|z-z_{k}\right| \geqq \alpha$ for each $k$. It is then clear that

$$
\inf \{|f(z)|:|z|=r\} \geqq \delta\left(\min _{k=1, \cdots, n}|r-| z_{k}||\right)
$$

whenever $0<r<\rho$ and $|r-| z_{k}||>0$ for each $k$.

Let $U$ be an open subset of $C$. A path in $U$ is a uniformly continuous mapping $\gamma$ of $[0,1]$ into $U$ with $\gamma([0,1])^{-} \subset \subset U$. We say that $U$ is connected if, to each pair of distinct points $z, z^{\prime}$ in $U$, there corresponds a path $\gamma$ in $U$ with $\gamma(0)=z, \gamma(1)=z^{\prime}$.

THEOREM 3. Let $f$ be analytic and not identically zero on the connected open set $U, \zeta \in U, r>0$ and $\bar{B}(\zeta, r) \subset \subset U$. Then $\inf \{|f(z)|$ : $|z-\zeta|=\rho\}>0$ for all but finitely many $\rho$ with $0<\rho<r$.

Proof. In view of Proposition 1, it will suffice to prove that $f$ is not identically zero on $\bar{B}(\zeta, r)$. To this end, choose $z_{0}$ in $U$ with $\left|f\left(z_{0}\right)\right|>0$, and construct a path $\gamma$ in $U$ with $\gamma(0)=z_{0}$ and $\gamma(1)=\zeta$. Compute in turn $\delta$ so that $0<\delta<r$ and $\{z \in C: d(z, \gamma([0,1])) \leqq$ $\delta\} \subset U$, and numbers $0=t_{0}<t_{1}<\cdots<t_{n}=1$ such that $\mid \gamma\left(t_{k+1}\right)-$ $\gamma\left(t_{k}\right) \mid<2 \delta$ for each $k$. Then $B\left(\gamma\left(t_{k+1}\right), \delta\right)$ intersects $B\left(\gamma\left(t_{k}\right), \delta\right)$; so that, by Proposition 1 , if $f$ is not identically zero on $\bar{B}\left(\gamma\left(t_{k}\right), \delta\right)$, then it is not identically zero on $\bar{B}\left(\gamma\left(t_{k+1}\right), \delta\right)$. As $\left|f\left(z_{0}\right)\right|>0$, it follows that $f$ is not identically zero on $\bar{B}(\zeta, \delta) \subset \bar{B}(\zeta, r)$.

THEOREM 4. Let $f$ be analytic and not identically zero on the connected open set $U$. Let $K$ be a compact set well contained in $U$, and $\varepsilon>0$. Then either inf $\{|f(z)|: z \in K\}>0$ or there exist finitely many points $z_{1}, \cdots, z_{n}$ of $U$ and an analytic function $g$ on $U$ such that

$$
f(z)=\left(z-z_{1}\right) \cdots\left(z-z_{n}\right) g(z) \quad(z \in U),
$$

$\inf \{|g(z)|: z \in K\}>0$, and $d\left(z_{k}, K\right)<\varepsilon$ for each $k$.

Proof. With $\delta$ chosen so that $0<2 \delta<\varepsilon$ and $\{z \in C: d(z, K) \leqq$ $2 \delta\} \subset U$, let $\left\{\zeta_{1}, \cdots, \zeta_{\nu}\right\}$ be a $\delta$-net of $K$. It will suffice to prove that there exist analytic functions $f_{0}=f, f_{1}, \cdots, f_{\nu}$ on $U$ such that, for each $k \in\{1, \cdots, \nu\}$,

and

(i) $\quad \inf \left\{\left|f_{k}(z)\right|: z \in \bigcup_{j=1}^{k} \bar{B}\left(\zeta_{j}, \delta\right)\right\}>0$

(ii) either $f_{k}=f$ or there exist finitely many points $z_{1}, \cdots, z_{n(k)}$ of $U$ such that

$$
f(z)=\left(z-z_{1}\right) \cdots\left(z-z_{n(k)}\right) f_{k}(z)
$$


for each $z$ in $U$.

Suppose that $0 \leqq k<\nu$ and that we have found $f_{k}$. By Theorem 3, there exists $r$ such that $\delta<r<2 \delta$ and inf $\left\{|f(z)|:\left|z-\zeta_{k+1}\right|=r\right\}>0$. By Theorem 1, either inf $\left\{|f(z)|:\left|z-\zeta_{k+1}\right| \leqq r\right\}>0$, in which case we set $f_{k+1}=f_{k}$; or, as we may now assume, there exist finitely many points $x_{1}, \cdots, x_{m}$ of $B\left(\zeta_{k+1}, r\right)$ and a differentiable function $g$ on $\bar{B}\left(\zeta_{k+1}, r\right)$ such that

$$
f_{k}(z)=\left(z-x_{1}\right) \cdots\left(z-x_{m}\right) g(z) \quad\left(z \in \bar{B}\left(\zeta_{k+1}, r\right)\right)
$$

and $\inf \left\{|g(z)|:\left|z-\zeta_{k+1}\right| \leqq r\right\}>0$. It follows from [1, p. 132, Cor. 4] that there exists an analytic function $f_{k+1}$ on $U$ such that

$$
f_{k}(z)=\left(z-x_{1}\right) \cdots\left(z-x_{m}\right) f_{k+1}(z) \quad(z \in U)
$$

and $g$ is the restriction of $f_{k+1}$ to $\bar{B}\left(\zeta_{k+1}, r\right)$. Our inductive assumptions about $f_{k}$ now ensure that $f_{k+1}$ also satisfies (i) and (ii). This completes our induction.

Another path to Theorem 4 has been taken by Orevkov [5], who uses [1, p. 136, Lemma 8] to estimate the number of zeroes of $f$ in the region of interest. It is worth noting, and not difficult to show, that the estimates given by Theorem 2 above are much more efficient than those embodied in [1, p. 136, Lemma 8].

Note that the only application of the connectivity of $U$ in the above proof of Theorem 4 is in the construction of $r$ such that inf $\left\{|f(z)|:\left|z-\zeta_{k+1}\right|=r\right\}>0$. It follows from Proposition 1 that the conclusion of Theorem 4 will hold even if $U$ is not connected, provided that $f$ does not vanish on some dense subset of $K$. With this remark in mind, we can now derive our final results.

THEOREM 5. Let $f$ be analytic on the open set $U$, and let $K$ be a compact set well contained in $U$ on which $f$ does not vanish. Then $\inf \{|f(z)|: z \in K\}>0$, and $1 / f$ is differentiable on $K$.

Proof. Either $\inf \{|f(z)|: z \in K\}>0$ or there exist finitely many points $z_{1}, \cdots, z_{n}$ of $U$ and an analytic function $g$ on $U$ such that

$$
f(z)=\left(z-z_{1}\right) \cdots\left(z-z_{n}\right) g(z) \quad(z \in U)
$$

and $0<m=\inf \{|g(z)|: z \in K\}$. In the latter case, for each $z$ in $K$ and each $j$, we have $\left|z-z_{j}\right|>0$; whence [2, p. 29, 2.2]

$$
0<d=\min _{j=1, \cdots, n} d\left(z_{j}, K\right) .
$$

Thus $|f(z)|>d^{n} m$ for each $z$ in $K$. Then desired conclusions follow immediately. 
COROLLARY. Let $f$ be analytic and nonvanishing on the open set $U$. Then $1 / f$ is analytic on $U$.

ACKNowledgment. This paper was written while the author was visiting the Department of Mathematics at New Mexico State University. The author is grateful to Allan Calder, William Julian, Ray Mines and Fred Richman for their contributions to the discussions which led to Theorems 3-5. He is particularly grateful to the last-named for pointing out an error in the original version of the proof of Theorem 2.

\section{REFERENCES}

1. Errett Bishop, Foundations of Constructive Analysis, McGraw-Hill, New York, 1967.

2. D. S. Bridges, Constructive Functional Analysis, Research Notes in Mathematics, No. 28, Pitman, London, 1979.

3. - A criterion for compactness in metric spaces?, Z. Math. Logik Grund. Math. 25 (1979), 97-98.

4. Allan Calder, Constructive Mathematics, Scientific American, October 1979, 146-172.

5. V. P. Orevkov, New proof of the uniqueness theorem for constructively differentiable functions of a complex variable, J. Soviet Math., 8, No. 3, (Sept. 1977), 329-334.

6. E. G. Phillips, Some Topics in Complex Analysis, Pergamon Press, Oxford, 1966.

7. E. C. Titchmarsh, The Theory of Functions, Clarendon Press, Oxford, 1932.

Received November 30, 1979 and in revised form March 14, 1980.

University College

Buckingham MK18 1EG, England 


\section{PACIFIC JOURNAL OF MATHEMATICS}

\section{EDITORS}

DONALD BABBITT (Managing Editor)

University of California

Los Angeles, California 90024

Hugo RossI

University of Utah

Salt Lake City, UT 84112

C. C. MOORE and ANDREW OGG

University of California

Berkeley, CA 94720

\section{J. DugundJI}

Department of Mathematics University of Southern California Los Angeles, California 90007

R. FinN and J. Milgram Stanford University Stanford, California 94305

\section{ASSOCIATE EDITORS}
R. ARENS
E. F. BECKENBACH
B. H. Neumann
F. WOLF
K. YoshidA

\section{SUPPORTING INSTITUTIONS}

UNIVERSITY OF ARIZONA

UNIVERSITY OF BRITISH COLUMBIA

CALIFORNIA INSTITUTE OF TECHNOLOGY

UNIVERSITY OF CALIFORNIA

MONTANA STATE UNIVERSITY

UNIVERSITY OF NEVADA, RENO

NEW MEXICO STATE UNIVERSITY

OREGON STATE UNIVERSITY
UNIVERSITY OF OREGON

UNIVERSITY OF SOUTHERN CALIFORNIA

STANFORD UNIVERSITY

UNIVERSITY OF HAWAII

UNIVERSITY OF TOKYO

UNIVERSITY OF UTAH

WASHINGTON STATE UNIVERSITY

UNIVERSITY OF WASHINGTON 


\section{Pacific Journal of Mathematics}

\section{Vol. 96, No. $1 \quad$ November, 1981}

Hédi Amara, Groupe des classes et unité fondamentale des extensions quadratiques relatives à un corps quadratique imaginaire principal $\ldots \ldots \ldots 1$

Douglas S. Bridges, On the isolation of zeroes of an analytic function $\ldots \ldots 13$ Andrew J. Casson and John L. Harer, Some homology lens spaces which

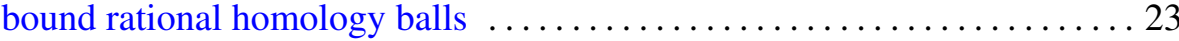

Z. A. Chanturia, On the absolute convergence of Fourier series of the classes $H^{\omega} \cap V[v]$

J.-F. Colombeau and Mário Carvalho Matos, On some spaces of entire functions defined on infinite-dimensional spaces $\ldots \ldots \ldots \ldots \ldots \ldots \ldots 63$

Edwin Duda, Pointwise periodic homeomorphisms on chainable continua . . .77

Richard F. Gustafson, A simple genus one knot with incompressible spanning surfaces of arbitrarily high genus $\ldots \ldots \ldots \ldots \ldots \ldots \ldots \ldots 1$

Fumio Hiai, Masanori Ohya and Makoto Tsukada, Sufficiency, KMS condition and relative entropy in von Neumann algebras

Ted Hurley, Intersections of terms of polycentral series of free groups and free Lie algebras. II .................................. 111

Robert Edward Jamison, II, Partition numbers for trees and ordered sets . . 115 R. D. Ketkar and N. Vanaja, A note on FR-perfect modules ............. 141 Michihiko Kikkawa, On Killing-Ricci forms of Lie triple algebras ....... 153

Jorge Lewowicz, Invariant manifolds for regular points 163

Richard W. Marsh, William H. Mills, Robert L. Ward, Howard Rumsey and Lloyd Richard Welch, Round trinomials .....

Claude Schochet, Topological methods for $C^{*}$-algebras. I. Spectral sequences

Yong Sian So, Polynomial near-fields?

Douglas Wayne Townsend, Imaginary values of meromorphic functions in the disk

Kiyoshi Watanabe, Coverings of a projective algebraic manifold .. 243

Martin Michael Zuckerman, Choosing $l$-element subsets of $n$-element sets 